\title{
Antigenic diversity in flagellar epitopes among Bacillus piliformis isolates
}

\author{
G. P. BOIVIN, R. R. HOOK* and L. K. RILEY \\ Department of Veterinary Pathology, W213 Veterinary Medicine, College of Veterinary Medicine, University of \\ Missouri-Columbia, Columbia, MO 65211, USA
}

\begin{abstract}
Summary. Monoclonal antibodies (MAbs) were developed to Bacillus piliformis isolatespecific flagellar epitopes and used to group $B$. piliformis isolates on the basis of epitope expression. BALB/c mice immunised with flagella purified from various $B$. piliformis isolates served as the source of immune spleen cells for fusion with SP2/0Ag14 myeloma cells. Evaluation of hybridoma culture medium by ELISA against various bacterial species and $B$. piliformis isolates indicated that 482 of 2127 hybridomas secreted antibodies specific for $B$. piliformis. Specificity of MAbs for flagellar epitopes was demonstrated by indirect fluorescent antibody assays and Western blot analyses. Probing of $10 \mathrm{~B}$. piliformis isolates with MAbs indicated that four B. piliformis isolates each possessed a distinct and isolate-specific flagellar epitope; five other isolates shared a commun flagellar epitope. One isolate did not react with any of the MAbs specific for flagellar epitopes. Thus, B. piliformis isolates could be grouped into six antigenically distinct groups based upon flagellar epitope expression. Additionally, a $\mathrm{MAb}$ reactive with a cell-associated component recognised all but one isolate. This serological grouping of $B$. piliformis isolates agrees with the grouping of isolates based upon genetic and physiological characteristics, and supports the assertion that there are different strains among B. piliformis isolates.
\end{abstract}

\section{Introduction}

Bacillus piliformis, the aetiological agent of Tyzzer's disease, is an unclassified, large, motile, gram-negative, obligately intracellular, spore-forming bacterium. Since the original observation of the organism in mice by Tyzzer in $1917,{ }^{1}$ B. piliformis has been identified in the gastrointestinal tract and liver of various laboratory and domestic animals, including mice, rats, hamsters, gerbils, rabbits, rhesus monkeys, dogs and several other species. ${ }^{2}$ Physiological criteria used in the classification of bacteria cannot be used with $B$. piliformis because this organism cannot be propagated on cell-free media. ${ }^{3}$ Thus, routine classification of an organism as B. piliformis is based solely on morphological and pathological characteristics.

Evidence of strain differences among $B$. piliformis isolates was first suggested in the early 1970 s by experimental pathogenicity and vaccination studies in mice. ${ }^{4-6}$ Host specificity and protection afforded by vaccination was most complete when the challenge isolate of $B$. piliformis was the same as the isolate used for vaccination. Further evidence of diversity among the various $B$. piliformis isolates was demonstrated by agar gel diffusion, complement fixation tests and
Western blot analyses. These tests revealed that both conserved and isolate-specific antigens were present in the various $B$. piliformis isolates. ${ }^{4,7}$ Subsequent studies by Motzel and Riley ${ }^{8}$ suggested that $B$. piliformis flagella expressed both conserved and isolate-specific epitopes.

Further evidence of antigenic diversity among $B$. piliformis isolates was provided by Toriumi et al.$^{9}$ who demonstrated that a monoclonal antibody (MAb), developed to a flagellar epitope of a $B$. piliformis isolate of rat origin, reacted with only three of seven $B$. piliformis isolates. In addition to the antigenic variability demonstrated by the above studies, investigations by Riley et al. ${ }^{7}$ demonstrated heterogeneity in morphological and physiological characteristics among $10 \mathrm{~B}$. piliformis isolates.

The studies cited above indicated that isolates of $B$. piliformis represent a diverse group of organisms. Furthermore, these studies also suggested that $B$. piliformis isolates might be grouped antigenically on the basis of expression of isolate-specific flagellar epitopes. In this study, we report the development of MAbs reactive with isolate-specific flagellar epitopes and grouping of $B$. piliformis isolates based upon reactivity with these MAbs. 


\section{Materials and methods}

\section{Reagents and equipment}

Reagents and equipment used in this study were as follows: Dulbecco's Modified Eagle's Medium and polyethylene glycol 4000 (Sigma): Omnimixer homogeniser (Omni International. Waterbury. CT. USA): HL-1 Ventrex synthetic medium (Endotronics, Coon Rapids. MN. USA): Falcon 3912 assay plates (Becton Dickinson, Oxnard. CA, USA); peroxidase-labelled goat anti-mouse IgG and FITC-labelled donkey antimouse IgG (Jackson Immuno Research Labs. West Grove, PA. USA): ABTS, peroxidase-labelled goat anti-mouse IgA and tetramethyl benzidine substrate (Kirkegaard and Perry Laboratories, Gaithersburg. MD. USA): peroxidase-labelled goat anti-mouse IgM (Zymed, San Francisco, CA, USA): peroxidase-labelled goat anti-mouse IgG1, IgG2a, IgG2b and IgG3 (Fisher Biotec, Pittsburgh, PA, USA): mol. wt standards (BioRad Laboratories, Richmond, CA, USA); biotinylated horse anti-mouse IgG and peroxidaselabelled streptavidin (Vector Laboratories, Burlingame, CA, USA).

\section{Bacterial strains}

Ten $B$. piliformis isolates of equine (E), guinea pig (GP), rabbit (B), mouse (M), hamster $(\mathrm{H} 1, \mathrm{H} 2$ and $H 3)$, gerbil (G), and rat (RI and R2) origin were used in the study. The isolation and origin of isolates have been described previously. '

\section{Animals}

Female VAH BALB/c mice, 6-8 weeks old, free of clinical disease and infection with known pathogenic organisms, were obtained from the National Cancer Institute. Gerbils known to be free of infection with $B$. piliformis were obtained from a breeding colony at the University of Missouri. All experiments were conducted under an approved Animal Care and Use Protocol and animals were maintained in compliance with PHS policy.

\section{Antigen preparation}

$B$. piliformis isolates were grown and harvested as described by Riley et al.' Briefly, B. piliformis were propagated in established Buffalo rat liver (BRL) or mouse fibroblast (3T3) mammalian cell lines cultured in Dulbecco's Modified Eagle's Medium supplemented with fetal calf serum $5-10 \%$ and $2 \mathrm{~mm}$ L-glutamine at $37^{\circ} \mathrm{C}$ with $\mathrm{CO}_{2} 10 \%$. Infected cell monolayers were harvested, sonicated to release intracellular bacteria, centrifuged at $2000 \mathrm{~g}$ for $10 \mathrm{~min}$ at $4^{\circ} \mathrm{C}$ to pellet the mammalian cells, and the supernate was centrifuged at $10000 y$ for $30 \mathrm{~min}$ at $4^{\circ} \mathrm{C}$ to pellet $B$. piliformis. After washing once with $\mathrm{PBS}, \mathrm{pH} 7 \cdot 4, B$. piliformis organisms were used as antigen in ELISA, IFA and Western blot analyses. Isolated flagella were prepared as described previously ${ }^{\star}$ and used as immunogen for MAb development. Briefly, $B$. piliformis flagella were sheared in a homogeniser (Omnimixer) at speed 5 for $60 \mathrm{~s}$ and centrifuged at $12000 \mathrm{~g}$ for $30 \mathrm{~min}$ at $4^{\circ} \mathrm{C}$ to pellet intact organisms. Flagella were pelleted by centrifugation twice at $58000 \mathrm{~g}$ for $2 \mathrm{~h}$ at $4^{\circ} \mathrm{C}$, and resuspended in sterile $\mathrm{PBS}, \mathrm{pH} \mathrm{7.4}$. Protein concentrations were determined by the $\mathrm{BCA}$ method with bovine serum albumin as standards.

\section{Development of MAbs}

MAbs were produced by the procedure of St Groth and Scheidegger ${ }^{10}$ as previously described. ${ }^{11}$ Three female VAH BALB/c mice, 6-8 weeks old, were immunised by intravenous injection with $10 \mu \mathrm{g}$ of isolated flagella and used in three separate fusion experiments. One BALB/c mouse was inoculated with $B$. piliformis flagella pooled from isolates $\mathrm{R} 1, \mathrm{~B}$ and $\mathrm{E}$. A second mouse was inoculated with flagella pooled from isolates M, GP, $\mathrm{Hl}$ and $\mathrm{G}$, and a third mouse was inoculated with flagella from isolate M. At 4-6 weeks after primary injection, each mouse was given, by intravenous injection, $10 \mu \mathrm{g}$ of the same flagellar proteins as those used for primary immunisation. Spleens from immunised mice killed 4 days after the secondary injection served as the source of immune cells for hybridisation experiments.

Spleen cells were prepared aseptically by teasing the donor spleen in serum-free HL-1 Ventrex medium supplemented with $2 \mathrm{~mm}$ L-glutamate, penicillin and streptomycin (HL-1 medium). For fusion, $1.5 \times 10^{8}$ spleen cells were fused with $5 \times 10^{7}$ viable SP2/0Ag 14 mouse myeloma cells grown in HL-1 medium; polyethylene glycol 4000, 50\% in HL-1 medium, was used as the fusing agent. Fused cells in HL-1 medium supplemented with fetal calf serum $2 \%$, hypoxanthine, aminopterine and thymidine (HAT medium), were distributed into the wells of 1596 -well culture plates seeded $24 \mathrm{~h}$ earlier with $1000 \mathrm{BALB} / \mathrm{c}$ peritoneal macrophages/well in HAT medium. Cultures were fed at 7 days with $150 \mu$ of HAT medium; 10-20 days after fusion, medium from wells containing vigorously growing hybridomas were screened by ELISA as described below.

\section{ELISA}

The procedure used in this study followed the methodology described by our laboratories for evaluation of MAbs to Campylobacter spp. ${ }^{11}$ Initial screening of hybridoma culture medium was for reactivity with pooled isolates of $B$. piliformis air-dried on to Falcon 3912 assay plates at $1.5 \times 10^{7}$ organisms/well; spent $\mathrm{SP} 2 / 0$ culture medium served as negative control medium. Reactivity was detected with peroxidaselabelled, goat anti-mouse IgG (Fc-specific) diluted 1 in 5000 in blocking buffer as secondary antibody, and ABTS as chromophore. Hybridomas producing OD readings of $>0.20$ were considered to be positive reactors.

Hybridomas demonstrating reactivity with $B$. pili- 
formis were also tested by ELISA for reactivity with BRL cells, 3T3 cells and a mixture of Pseudomonas aeruginosa, Proteus vulgaris and B.cereus. Hybridomas demonstrating readings of $<0.03$ OD were considered to be negative reactors with these antigens. Hybridomas that demonstrated specificity for B. piliformis were expanded into 24-well culture plates, tested again for specificity, cloned by limiting dilution, and again tested for specificity.

\section{Isotyping of $M A b s$}

B. piliformis-sensitised assay plates were used to isotype MAbs as described previously. ${ }^{12}$ After incubation of hybridoma supernate with antigen-coated wells, bound antibodies were detected by incubation with peroxidase-labelled goat anti-mouse IgA, IgM, IgG1, IgG2a, IgG2b and IgG3. Incubations, colour development and plate reading were as described above.

\section{Indirect immunofluorescent antibody assay (IFA)}

Twelve-well glass slides were loaded $(10 \mu \mathrm{l} /$ well $)$ with individual $B$. piliformis isolates at $c .10^{6}$ cells $/ \mathrm{ml}$, air-dried at room temperature and fixed in cold acetone for $10 \mathrm{~min}$. Hybridoma supernate diluted in PBS, pH 7.4, containing Tween $200.05 \%$ and powdered non-fat dried milk $2.5 \%$ (blocking buffer) were added to individual wells and incubated for $30 \mathrm{~min}$ at room temperature in a humidified chamber. After three 5-min washes with PBS, pH 7.4, containing Tween $200.05 \%$ (wash buffer), FITC-labelled, donkey anti-mouse $\mathrm{IgG}$ (Fc-specific) diluted 1 in 80 in wash buffer was added to each well and incubated for $30 \mathrm{~min}$ at room temperature in a humidified chamber. After four 5-min washes with wash buffer and two 5-min washes with PBS, pH 7.4, the slides were mounted and examined for specific fluorescence. Negative controls consisted of $B$. piliformis incubated with spent SP2/0 medium and MAbs incubated with a mixture of $P$. aeruginosa, Pr. vulgaris and B. cereus.

Indirect immunofluorescent antibody assays were also performed on B. piliformis-infected BRL cells and specimens of caecum and liver collected from gerbils 5 days after oral infection with $10^{8}$ viable $B$. piliformis organisms ( $\mathrm{R} 1$ isolate). Infected BRL cells and formalin-fixed tissues embedded in paraffin, cut to $2 \mu \mathrm{m}$ sections, mounted on glass slides, and rehydrated with PBS were processed for IFA staining as described above. Non-infected gerbil caecum and liver and BRL cells incubated with spent SP2/0 medium served as negative controls. Sections ( $5 \mu \mathrm{m}$ ) adjacent to the IFA stained sections were examined after Warthin-Starry staining to confirm specificity of the MAbs for $B$. piliformis.

\section{$S D S-P A G E$}

To identify individual peptides and glycoproteins recognised by the MAbs, whole $B$. piliformis organisms were subjected to SDS-PAGE under reducing con- ditions on vertical slab gels, as described by Laemmli. ${ }^{12}$ Proteins were stacked with acrylamide $4.5 \% \mathrm{w} / \mathrm{v}$ and separated on acrylamide $8 \% \mathrm{w} / \mathrm{v}$. Mol. wt standards consisted of rabbit muscle phosphorylase $\mathrm{B}$, bovine serum albumin, hen egg white ovalbumin, bovine carbonic anhydrase B, soybean trypsin inhibitor and hen egg white lysozyme.

\section{Immunoblotting}

Components of B. piliformis separated by SDSPAGE were transferred on to nitrocellulose membranes by the method of Towbin. ${ }^{13}$ After membranes were blocked overnight at room temperature by incubation in blocking buffer, they were probed for reactivity with the MAbs and developed with biotinylated goat anti-mouse IgG and peroxidase-labelled streptavidin as described previously. ${ }^{7}$ Antibody reactive bands were visualised by incubation with tetramethyl benzidine substrate.

\section{Results}

\section{Monoclonal antibody reactivity}

During the course of three separate hybridisation experiments, 482 of 2127 hybridomas demonstrated reactivity with $B$. piliformis isolates; $<10 \%(32 / 482)$ of the MAbs reactive with $B$. piliformis demonstrated reactivity with $P$. aeruginosa, $P r$. vulgaris, $B$. cereus, BRL cells or 3T3 cells. Based upon preliminary studies, 24 hybridomas were selected for more extensive characterisation. Specificity testing of these MAbs by ELISA demonstrated no reactivity with various bacteria that included: Citrobacter freundii, Klebsiella pneumoniae, Acinetobacter calcoaceticus, Enterobacter aerogenes, Salmonella typhimurium, K. marcescens, Streptococcus faecalis. Staphylococcus epidermidis, Campylobacter jejuni, Pr. vulgaris, Escherichia coli, Vibrio fluvialis, Shigella sonnei and Ent. cloacae.

MAbs specific for B. piliformis demonstrated various reactivity patterns when tested by ELISA with the 10 $B$. piliformis isolates (table). MAbs with single isolate specificity were demonstrated for isolates $\mathrm{B}, \mathrm{E}, \mathrm{R} 1$ and $M$. Hybridomas secreting antibodies that recognised epitopes on more than one isolate exhibited two reactivity patterns. One group of MAbs reacted exclusively with isolates $\mathrm{GP}, \mathrm{H} 1, \mathrm{H} 2, \mathrm{H} 3$ and $\mathrm{G}$; a second group of MAbs reacted with all B. piliformis isolates except $B$ and a high culture passage of $\mathrm{R} 1$. Isolate $\mathrm{R} 2$ was unique in that it reacted only with the latter group of MAbs.

\section{Isotypimg}

The selected MAbs were further characterised by analyses for antibody isotype. All MAbs were of the IgG1, IgG2a or IgG2b isotype (table).

Indirect fuorescent antibody assays (IFA)

MAbs demonstrating specificity for B. piliformis by 
Table. Characterisation of MAbs

\begin{tabular}{llllc}
\hline MAb & $\begin{array}{c}\text { Isolate } \\
\text { specificity }\end{array}$ & Isotype & $\begin{array}{c}\text { Epitope } \\
\text { location }\end{array}$ & $\begin{array}{c}\text { Antigen } \\
\text { size (kDa) }\end{array}$ \\
\hline $903-13 \mathrm{Cl}$ & $\mathrm{R}$ 1 & IgG1 & Flagella & 54 \\
$903-13 \mathrm{D} 3$ & $\mathrm{E}$ & IgG2a & Flagella & 54 \\
$903-7 \mathrm{C} 12$ & $\mathrm{~B}$ & IgG2b & Flagella & 54 \\
$916-6 \mathrm{~A} 9$ & $\mathrm{M}$ & IgG2a & Flagella & 54 \\
$911-8 \mathrm{C} 5$ & G. GP, H1, H2, H3 & IgG1 & Flagella & 54 \\
$903-12 \mathrm{G} 9$ & R1. R2. E. M. G & IgG1 & Cell-associated & $*$ \\
& GP. H1. H2, H3t & & & \\
& & & &
\end{tabular}

* No definitive peptide identified

+ Not reactive with isolate $B$ and a late culture passage of R1.
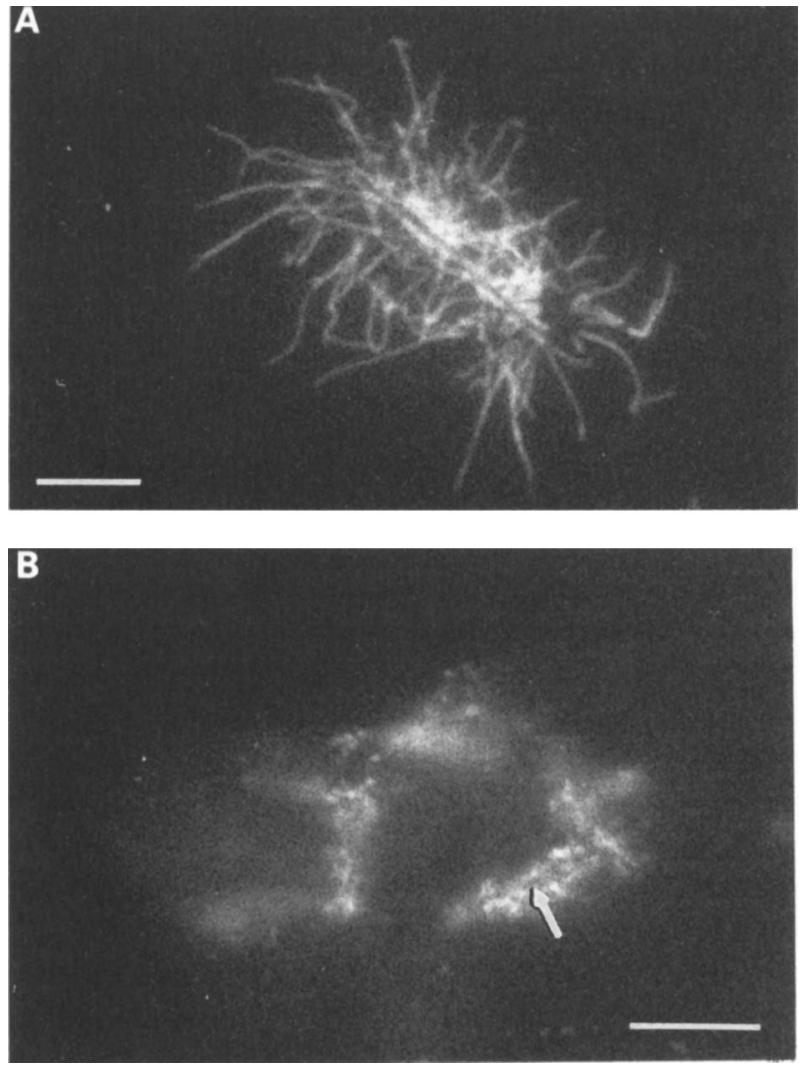

Fig. 1. IFA stains of B. piliformis isolate R1: A. with MAb 903-13CI demonstrating specificity for flagella--the photograph shows the bacteria as a dark non-staining rod surrounded by fluoresceinlabelled peritrichous flagella (bar, $5 \mu \mathrm{m}$ ): B. within an infected BRL cell probed with MAb 903-13CI-B. piliformis organisms show as dark non-staining rods (arrow) surrounded by clumped. fluoresceinlabelled flagella (bar, $8 \mu \mathrm{m}$ ).

ELISA were tested by IFA to determine the structural location of the epitope recognised and to confirm specificity of the antibodies. MAbs specific for $\boldsymbol{B}$. piliformis by ELISA demonstrated identical isolate and group specificities by IFA. Furthermore. IFA assays confirmed that antibodies did not react with other bacteria or with uninfected BRL and 3T3 cells. IFA tests also demonstrated that most of the $B$. piliformis-specific MAbs reacted with epitopes located on flagella. Fig. 1A demonstrates the reactivity of an $\mathrm{R} 1$-specific MAb, with flagella of $B$. piliformis isolate $\mathrm{R} 1$ from culture: MAbs reactive with other isolates demonstrated the same flagellar specificity (table). MAbs that demonstrated ELISA reactivity with all $B$.

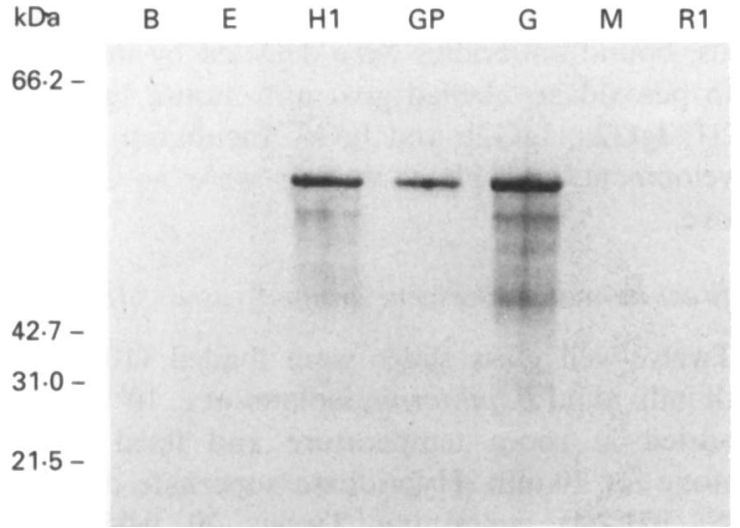

Fig. 2. Western blot of B. piliformis isolates B, E, H1, GP, G, M and $\mathrm{R} 1$ probed with MAb $911-8 \mathrm{C} 5$. Note the primary band at $c .54 \mathrm{kDa}$ defined with isolates GP, Hl and G. Lower mol. wt bands are believed to be break-down products of the 54-kDa protein. The approximate nigration of mol. wt markers is indicated.

piliformis isolates except $\mathrm{B}$ and the late culture passage of $R 1$ reacted with a cell-wall-associated epitope and showed no reactivity with flagella. Isolate $\mathrm{R} 2 \mathrm{did}$ not demonstrate any flagellar reactivity and reacted only with MAbs reactive with the cell-wall-associated epitope.

To confirm that the MAbs would recognise intracellular $B$. piliformis, and that the flagellar epitope(s) recognised was not a culture-induced antigen, MAbs specific for the R1 isolate were evaluated by IFA with $B$. piliformis-infected BRL cells and caecum and liver tissue sections from gerbils infected experimentally with the R1 isolate of $B$. piliformis. MAbs specific for the $\mathrm{R} 1$ isolate of $B$. piliformis demonstrated strong reactivity with isolate $\mathrm{Rl}$ flagella in both infected BRL cells (fig. 1B) and infected gerbil tissues (not shown). R1-specific MAbs did not show any specific fluorescence when tested by IFA with uninfected gerbil and liver tissue sections. MAbs specific for other $B$. piliformis isolates did not show any reactivity with the $R I$ isolate in tissue sections.

\section{Immunoblotting}

Immunoblots of $B$. piliformis components separated by SDS-PAGE and probed with the $B$. piliformisspecific antibodies confirmed the isolate and group specificity of the MAbs. MAbs that demonstrated flagellar reactivity by IFA reacted with epitopes 
located on a protein of c. $54 \mathrm{kDa}$. When a MAb specific for isolates $\mathrm{GP}, \mathrm{H} 1, \mathrm{H} 2, \mathrm{H} 3$ and $\mathrm{G}$ was used to probe Western blots of different $B$. piliformis isolates, specific immunoreactive bands were observed with isolates $\mathrm{G}, \mathrm{HI}$ and $\mathrm{GP}$, and were not present with isolates M, R1, E or B (fig. 2). Other MAbs that showed flagellar'specificity by IFA also reacted with a protein of c. $54 \mathrm{kDa}$ and confirmed specificities demonstrated by ELISA and IFA (table). Immunoblots probed with MAbs reactive with the $B$. piliformis cell-wall-associated epitope failed to develop any definitive band.

\section{Discussion}

In the present study, MAbs reactive with flagellar epitopes of $B$. piliformis were developed to nine of 10 isolates; none of the flagella-specific MAbs reacted with isolate R2, of rat origin. The specificity of the MAbs for B. piliformis was demonstrated by a lack of reactivity in ELISA against a wide variety of other bacterial species. Furthermore, specificity of the MAbs for flagellar epitopes of B. piliformis was demonstrated by IFA and Western blotting. IFA staining of liver and intestinal tissue sections from gerbils infected experimentally with isolate R1 further confirmed the specificity of the MAbs and demonstrated that the flagellar epitopes recognised by the antibodies were not cultureinduced epitopes.

Based upon our results, the isolates could be grouped into six serological groups with the flagellaspecific MAbs. In this grouping, isolates, B, E, M and $\mathrm{R} 1$ each possess a distinct flagellar epitope and represent four distinct serological groups. Isolates $\mathrm{G}$, GP, $\mathrm{H} 1, \mathrm{H} 2$ and $\mathrm{H} 3$ share a common flagellar epitope and represent a fifth serological group. Isolate $\mathrm{R} 2$ does not possess a flagellar epitope recognisable by any of our flagella-specific MAbs and represents the sixth serological group. These results are consistent with previous reports that suggested considerable heterogeneity among $B$. piliformis isolates. ${ }^{4-6,8,9}$

The grouping of $B$. piliformis isolates on the basis of expression of flagellar epitopes is in agreement with observations made in our laboratories on the growth and physiological characteristics of the isolates. For example, isolates GP, H1, H2, H3 and G, which demonstrate similar growth and sporulation rates in culture, ${ }^{7}$ are grouped into the same antigenic group by

\section{References}

1. Tyzzer EE. A fatal disease of the Japanese waltzing mouse caused by a spore-bearing bacillus (Bacillus piliformis $\mathrm{N}$. SP.). J Med Res 1917; 37: 307-388.

2. Fries AS. Studies on Tyzzer's disease : isolation and propagation of Bacillus piliformis. Lab An 1977; 11: 75-78.

3. Thunert A. Is it possible to cultivate the agent of Tyzzer's disease (Bacillus piliformis) in cellfree media? Z Ver 1984; 26: $145-150$.

4. Fujiwara $K$, Kurashina $H$, Magaribuchi $T$, Takenaka $S$, Yokoiyama S. Further observation on the difference
MAb 911-8C5. Also, at least three of the isolates in this group (GP, H1 and G) produce similar, if not identical, toxins. ${ }^{14}$ The observation that isolate $\mathrm{R} 2 \mathrm{did}$ not react with any of the MAbs specific for flagellar epitopes of the other B. piliformis isolates is also consistent with previous observations. The growth and physiological characteristics of this isolate differ from those of the other isolates, in that isolate $R 2$ is difficult to grow in the vegetative phase and shows a much higher degree of sporulation than do other isolates (unpublished data). These data suggest that at least one additional serological and physiological group is present in B. piliformis and is represented by isolate $\mathrm{R} 2$.

The observation that $B$. piliformis isolates express isolate-specific flagellar epitopes was not surprising, since previous studies suggested that $B$. piliformis possesses both conserved and isolate-specific antigens. ${ }^{4.7 .8}$ However, the failure to develop a MAb that reacted with a flagellar epitope common to all $B$. piliformis isolates was unexpected. A previous study by Toriumi et al. ${ }^{9}$ demonstrated that a MAb developed to an isolate of rat origin also reacted with an isolate of mouse origin and suggested the presence of a conserved flagellar epitope. A conserved flagellar epitope in B. piliformis was also suggested by the study of Motzel and Riley. ${ }^{8}$ One MAb developed in this current study reacted with all $B$. piliformis isolates except isolate B. However, IFA and Western blot analyses indicated that the epitope recognised by this antibody was associated with the cell and not with flagella. Our data also indicated that this $\mathrm{MAb}$ reacted with early culture passages of isolate $\mathrm{R} 1$ but failed to react with a late culture passage of $\mathrm{R} 1$. These data suggest that long-term culture in vitro of at least some $B$. piliformis isolates may result in the loss of this cell-wallassociated antigen.

Although bacterial flagella are best known for their role in motility, they also play an important role in pathogenesis and may function as virulence factors. ${ }^{18-18}$ Current studies in our laboratories are directed towards determining the relationship(s) among flagellar epitopes (serological groups), pathogenesis and host specificity in experimental and naturally-occurring $B$. piliformis infections.

This work was supported in part by a COR grant from the College of Veterinary Medicine, University of Missouri, by Public Health Service Grant RR04568, and by a commercial grant from Charles River Laboratories. We appreciate the technical assistance of Ms Mona Garro, and photographic support of H. Wilson.

between Tyzzer's organisms from mice and those from rats. Jpn J Exp Med 1973; 43: 307-315.

5. Fujiwara K, Takasaki Y, Kubokawa K, Takenaka S, Kubo M, Sato K. Pathogenic and antigenic properties of the Tyzzer's organisms from feline and hamster cases. Jpn J Exp Med $1974 ; 44: 365-372$.

6. Fujiwara K, Yamada A, Ogawa H, Oshima Y. Comparative studies on the Tyzzer's organisms from rats and mice. $J p n$ $J$ Exp Med 1971; 41 : 125-133.

7. Riley LK, Besch-Williford C, Waggie KS. Protein and antigenic heterogeneity among isolates of Bacillus piliformis. Infect Immun 1990; 58: 1010-1016. 
8. Motzel SL. Riley LK. Bacillus piliformis flagellar antigens for serodiagnosis of Tyzzer's disease. J Clin Microbiol 1991: 29: $2566-2570$.

9. Toriumi W. Okada N. Kawamura S. Fujiwara K. Production and characterization of monoclonal antibodies to Tyzzer's organism (Bacillus piliformis). Jpn J I'et Sci 1986:48: 977988 .

10. St Groth SF. Scheidegger D. Production of monoclonal antibodies: strategy and tactics. $J$ Immmol Methods 1980 : 35: $1-21$.

11. Stills HF. Hook RR, Sprouse RF. Utilization of monoclonal antibodies to evaluate the involvement of Campllohacter jejuni in proliferative ileitis in Syrian hamsters (Mesocricetis auratus). Infect Immun 1987: 55: 2240-2246.

12. Laemmli UK. Cleavage of structural proteins during the assembly of the head of bacteriophage T4. Nature 1970: 227: $680-685$.
13. Towbin H. Staehelin T, Gordon J. Electrophoretic transfer of proteins from polyacrylamide gels to nitrocellulose sheets: procedure and some applications. Proc Natl Acad Sci USA 1983: 76: $4350-4354$.

14. Riley LK. Caffrey CJ, Musille VS, Meyer JK. Cytotoxicity of Bacillus piliformis. J Med Microbiol. 1992; 37: 77-80.

15. Attridge SR. Rowley D. The role of flagellum in the adherence of Vibrio cholerae. J Infect Dis 1983; 147: 864-872.

16. Carsiotis M, Weinstein DL, Karch H. Holder IA, O'Brien AD. Flagella of Salmonella typhimurium are a virulence factor in infected C57BL/6J mice. Infect Immun 1984: 46: $814-818$.

17. Guentzel MN. Berry LJ. Motility as a virulence factor for Vibrio cholerae. Infect Immun 1975; 11: 890-897.

18. Weinstein DL, Carsiotis M. Lissner CR, O'Brien AD. Flagella help Salmonella typhimurium survive within murine macrophages. Infect Immun 1984; 46: 819 825 . 